\title{
Global-in-Time Domain Decomposition for a Nonlinear Diffusion Problem
}

Elyes Ahmed and Caroline Japhet and Michel Kern

\section{Introduction}

We study a simplified model for two-phase flow in porous media, where the medium is made of two (or more) different rock types. Each rock type is a subdomain with a distinct capillary pressure function so that the saturation becomes discontinuous across the interface between the different regions. This leads to the phenomenon of capillary trapping (see [14], or [5]).

In this paper we develop a non-overlapping domain decomposition method that combines the Optimized Schwarz Waveform Relaxation method with Robin transmission conditions and the discontinuous Galerkin method in time. The domain decomposition method we present is global-in-time, which provides flexibility for using non-matching time grids so as to handle the very different time scales that occur in the different rocks of the porous medium. The method is a generalization of previous work on linear diffusion or diffusion-advection problems [10, 11].

We state briefly the physical model, referring to [2] for further details. The porous medium $\Omega$ is heterogeneous and made-up of two rock types, represented by open bounded subsets $\left(\Omega_{i}\right)_{i \in\{1,2\}}$ (the restriction to two subdomain is only to simplify the exposition, and indeed the example given in section 4 uses more than 2 subdomains). The subdomains share the interface $\Gamma=\bar{\Omega}_{1} \cap \bar{\Omega}_{2}$. We suppose that each subdomain

Elyes Ahmed

Inria, 2 rue Simone iff, 75589 Paris, France,

current address Department of Mathematics, University of Bergen, P. O. Box 7800, N-5020 Bergen, Norway, e-mail: Elyes.Ahmed@uib.no

Caroline Japhet

Université Paris 13, Sorbonne Paris Cité, LAGA, CNRS(UMR 7539), 93430, Villetaneuse, France, e-mail: japhet@math.univ-paris13.fr

Michel Kern

Inria, 2 rue Simone iff, 75589 Paris, France, e-mail:michel.kern@inria.fr

Université Paris-Est, CERMICS (ENPC), 77455 Marne-la-Vallée 2, France. 
$\Omega_{i}$ is homogeneous, so that the physical properties depend on space only through the subdomain index.

We consider the following nonlinear diffusion problem (for some time $T>0$ )

$$
\partial_{t} u_{i}-\nabla \cdot\left(\lambda_{i}\left(u_{i}\right) \nabla \pi_{i}\left(u_{i}\right)\right)=0, \quad \text { in } \Omega_{i} \times(0, T),
$$

for scalar unknowns $u_{i}=u_{\mid \Omega_{i}} \in[0,1]$ representing the gas saturation. This model can be obtained from the complete two-phase flow model by neglecting the advection terms in the saturation equation, so that the saturation and pressure equations become completely decoupled (see [5] for details). In the above reference, it is shown that this simplified model is sufficient to study gas trapping in low capillary pressure regions. The functions $\pi_{i}\left(u_{i}\right):[0,1] \rightarrow \mathbb{R}$ (Lipschitz and strictly increasing) and $\lambda_{i}\left(u_{i}\right):[0,1] \rightarrow \mathbb{R}$ are respectively the capillary pressure and the global mobility of the gas in subdomain $\Omega_{i}$. Initial data $u_{0} \in L^{2}([0,1])$ is given with $u_{0}>0$, and for simplicity we assume homogeneous Neumann boundary conditions on $\partial \Omega$.

Transmission conditions across the interface $\Gamma \times[0, T]$ are needed to complement (17) (see [6]). In the simple case where $\pi_{1}(0)=\pi_{2}(0)$ and $\pi(1)=\pi_{2}(1)$, the transmission conditions are simply the continuity of the capillary pressure and of the diffusive flux across the interface:

$$
\begin{aligned}
\pi_{1}\left(u_{1}\right) & =\pi_{2}\left(u_{2}\right) \\
\lambda_{1} \nabla \pi_{1}\left(u_{1}\right) \cdot \mathbf{n}_{1} & =-\lambda_{2} \nabla \pi_{2}\left(u_{2}\right) \cdot \mathbf{n}_{2}
\end{aligned} \text { on } \Gamma \times[0, T],
$$

where $\mathbf{n}_{i}$ is the unit, outward pointing, normal vector field on $\partial \Omega_{i}$. In the case when the above matching conditions are not satisfied, one has to truncate the capillary pressure functions and the transmission conditions are given in terms of the modified functions (see [1, 2] for details).

In the next section, this physical problem is rewritten in a form better suited for mathematical and numerical analysis. In particular, the existence of a weak solution of the local Robin problems is addressed. A semi-discrete formulation based on discontinuous Galerkin in time is given in section 3 and numerical experiments using a finite volume method are described in section 4

\section{Space-time domain decomposition at the continuous level}

The model stated above is well adapted to physical modeling, but is difficult to handle mathematically because of the low regularity of the solutions. To obtain mathematical results, it has been found useful to introduce the Kirchhoff transformation [5], so that $\lambda_{i}$ and $\pi_{i}$ are replaced by a single function $\varphi_{i}$, and following [4] to introduce new functions $\left(\Pi_{i}\right)_{i=1,2}$ that are smoother than $\pi_{i}$ but satisfy

$$
\pi_{1}\left(u_{1}\right)=\pi_{2}\left(u_{2}\right) \Leftrightarrow \Pi_{1}\left(u_{1}\right)=\Pi_{2}\left(u_{2}\right) .
$$

In terms of the new functions, the problem becomes 


$$
\partial_{t} u_{i}-\Delta \varphi_{i}\left(u_{i}\right)=0, \quad \text { in } \Omega_{i} \times(0, T), \quad u_{i}(\cdot, 0)=u_{0}, \quad \text { in } \Omega_{i},
$$

together with a Neumann boundary condition on $\partial \Omega_{i} \backslash \Gamma$ and the transmission conditions

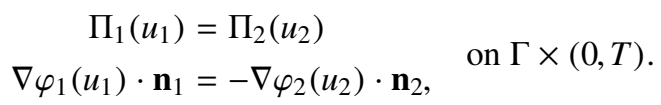

An existence theorem is known for the transmission problem (3), 44, see [4, 5] where existence of a suitably defined weak solution is proved.

An equivalent formulation to the model problem (3)-(4) can be obtained by replacing (4) by equivalent Robin transmission conditions on $\Gamma \times(0, T)$ :

$$
\begin{aligned}
& \nabla \varphi_{1}\left(u_{1}\right) \cdot \mathbf{n}_{1}+\alpha_{1} \Pi_{1}\left(u_{1}\right)=-\nabla \varphi_{2}\left(u_{2}\right) \cdot \mathbf{n}_{2}+\alpha_{1} \Pi_{2}\left(u_{2}\right), \\
& \nabla \varphi_{2}\left(u_{2}\right) \cdot \mathbf{n}_{2}+\alpha_{2} \Pi_{2}\left(u_{2}\right)=-\nabla \varphi_{1}\left(u_{1}\right) \cdot \mathbf{n}_{1}+\alpha_{2} \Pi_{1}\left(u_{1}\right),
\end{aligned}
$$

where $\alpha_{1}$ and $\alpha_{2}$ are two functions on $\Gamma \times(0, T)$ that can be chosen to enhance the convergence of the method (see [9, 10] for linear problems and [3] for a reactiondiffusion problem with nonlinear source term). It is shown in [1] how the Robin transmission conditions can be extended to Ventcell transmission conditions, to further improve the convergence of the method.

The Optimized Schwarz Waveform Relaxation with nonlinear Robin transmission conditions (NL-OSWR) is defined by the following iterations, where $\Psi_{i}^{0}$ is a given initial Robin guess on $\Gamma \times(0, T)$ for $i=1,2$ :

$$
\begin{aligned}
\partial_{t} u_{i}^{k}-\Delta \varphi_{i}\left(u_{i}^{k}\right) & =0, & & \text { in } \Omega_{i} \times(0, T), \\
\nabla \varphi_{i}\left(u_{i}^{k}\right) \cdot \mathbf{n}_{i}+\alpha_{i} \Pi_{i}\left(u_{i}^{k}\right) & =\Psi_{i}^{k-1}, & & \text { on } \Gamma \times(0, T),
\end{aligned}
$$

with suitable initial and boundary conditions, then set

$$
\Psi_{i}^{k}:=-\nabla \varphi_{j}\left(u_{j}^{k}\right) \cdot \mathbf{n}_{j}+\alpha_{i} \Pi_{j}\left(u_{j}^{k}\right), \quad j=(3-i), k \geq 1 .
$$

We give an existence result for the subdomain problem, namely problem [6 with the iteration $k$ and the subdomain $\Omega_{i}$ fixed. This is needed because of the Robin boundary condition. First a notion of weak solution is defined:

Definition 1 (Weak solution for the local Robin problem) A function $u$ is said to be a weak solution of problem (6) (with initial condition $u_{0}$ and homogeneous Neumann boundary condition on $\partial \Omega_{i} \backslash \Gamma$ if it satisfies:

1. $u \in L^{\infty}\left(\Omega_{i} \times(0, T)\right), \quad 0 \leq u \leq 1$ a.e. in $\Omega_{i} \times(0, T)$,

2. $\varphi(u) \in L^{2}\left(0, T ; H^{1}\left(\Omega_{i}\right)\right)$, and $\Pi(u, \cdot) \in L^{2}\left(0, T, H^{1}\left(\Omega_{i}\right)\right)$,

3. For all $\psi \in C_{\text {test }}=\left\{h \in H^{1}(\Omega \times(0, T)), h(., T)=0\right\}$, 


$$
\begin{aligned}
&-\int_{0}^{T} \int_{\Omega_{i}} u(\mathbf{x}, t) \partial_{t} \psi(\mathbf{x}, t) \mathrm{d} \mathbf{x} \mathrm{d} t-\int_{\Omega_{i}} u_{0} \psi(\mathbf{x}, 0) \mathrm{d} \mathbf{x} \\
&+\int_{0}^{T} \int_{\Omega_{i}} \nabla \varphi(u(\mathbf{x}, t)) \cdot \nabla \psi(\mathbf{x}, t) \mathrm{d} \mathbf{x} \mathrm{d} t-\int_{0}^{T} \int_{\Gamma} \alpha \Pi(u(\mathbf{x}, t)) \psi \mathrm{d} \gamma(\mathbf{x}) \mathrm{d} t \\
&=\int_{0}^{T} \int_{\Gamma} \Psi_{i}^{k-1}(\mathbf{x}, t) \psi \mathrm{d} \gamma(\mathbf{x}) \mathrm{d} t,
\end{aligned}
$$

where $\mathrm{d} \gamma(\mathbf{x})$ is the $(d-1)$-dimensional Lebesgue measure on $\partial \Omega_{i}$.

The following existence result is proven in [2].

Theorem 1 Assume that the initial condition $u_{0 i}=u_{0 \mid \Omega_{i}}$ is in $L^{\infty}\left(\Omega_{i}\right)$ and satisfies $u_{0 i}(x) \in[0,1]$ for all $x \in \Omega_{i}$, that $\Psi_{i}^{k-1} \in L^{2}\left(\Omega_{i} \times(0, T)\right)$, that the following conditions hold:

1. $\varphi_{i}$ is a strictly increasing Lipschitz continuous function on $(0,1)$;

2. $\Pi_{i}$ is a non-decreasing continuous function on $(0,1)$;

and that $\alpha_{i}$ is chosen such that holds:

$$
0<\Psi_{i}^{k}(x, t)<\alpha_{i} \Pi(1), \forall(x, t) \in \Omega_{i} \times(0, T) .
$$

Then there exists a weak solution to Problem (6) in the sense of Definition 1

The proof is an adaptation to Robin boundary conditions of the proof in [4, 5]. It is based on the convergence of a finite volume scheme.

We have chosen to state assumptions 1) and 2) above in terms of the functions $\varphi_{i}$ and $\Pi_{i}$, but of course one can translate the regularity conditions in terms of (essentially the same) conditions on the original functions $\pi_{i}$ and $\lambda_{i}$, as stated in the introduction. Additionally, note that in the context of the NL-OSWR method assumption (9) will have to be checked iteratively to prove that the algorithm is well posed (see section 3).

\section{Semi-discrete space-time domain decomposition with different time steps in the subdomains}

In order to focus on the non-conforming in time discretization, we keep the problem continuous in space.

We introduce a non-conforming time discretization, that is each subdomain $\Omega_{i}$ has its own time discretization, by using a (lowest order) Discontinuous Galerkin (DG) time discretization on each subdomain, together with a projection across the interface (see [9, 10] for an analysis in the linear case). More precisely, for integers $M_{i}$, define $\delta t_{i}=T / M_{i}+1$, and and partition $[0, T]$ in sub-intervals $J_{i}^{n}=\left(t_{i}^{n}, t_{i}^{n+1}\right]$ of size $\delta t_{i}$, where $t_{i}^{n}=n \delta t_{i}$, for $n=0, \ldots, M_{i}+1$. The time grid is denoted by $\mathcal{M}_{i}=\left\{J_{i}^{n}, n=0, \ldots, M_{i}\right\}$. 
In the NL-OSWR method, we have to solve problem (6) in each subdomain over the whole time interval $(0, T)$ (or over a time window). In order to deal with the non-conformity in time, it will be convenient to regard the discrete unknowns as piece-wise constant functions in time. For each $i=1,2$, we let

$$
u_{i}^{k}(t)=u_{i}^{k, n} \text { for } t \in J_{i}^{n} .
$$

where $u_{i}^{k, n}$ is the semi-discrete counterpart of $u_{i}$ on $J_{i}^{n}$. Given initial iterates $\Psi_{i}^{0}(t)$, for $i=1,2$, we describe one iteration of the NL-OSWR method.

Starting from the semi-discrete initial condition $u_{i}^{k, 0}=u_{0 i}$, a semi-discrete solution $\left(u_{i}^{k, n+1}\right)_{i=1,2}$ at time step $n \in\left\{0, \ldots, M_{i}\right\}$ is computed by solving

$$
\begin{array}{clrl}
\frac{u_{i}^{k, n+1}-u_{i}^{k, n}}{\delta t_{i}}-\Delta \varphi_{i}\left(u_{i}^{k, n+1}\right) & =0 & & \text { in } \Omega_{i}, \\
\nabla \varphi_{i}\left(u_{i}^{k, n+1}\right) \cdot \mathbf{n}_{i}+\alpha_{i} \Pi_{i}\left(u_{i}^{k, n+1}\right) & =\frac{1}{\delta t_{i}} \int_{J_{i}^{n}} \Psi_{i}^{k-1}(t) d t, & & \text { on } \Gamma \times(0, T),
\end{array}
$$

We must now define $\Psi_{i}^{k}(t)$ for the next NL-OSWR iteration. As we do not assume matching time grids across the space-time interface $\Gamma \times(0, T)$, data must be transferred from one space-time subdomain to its neighbor. This is done by using a suitable $L^{2}$ projection. Thus we define, for $n=1, \ldots, M_{i}$, with $j=3-i$,

$$
\left(\Psi_{i}^{k}(t)\right)_{\mid J_{i}^{n}}=\frac{1}{\delta t_{i}} \sum_{m=1}^{M_{j}} \int_{J_{i}^{n} \cap J_{j}^{m}}\left(-\nabla \varphi_{j}\left(u_{j}^{k-1}(t)\right) \cdot \mathbf{n}_{j}+\alpha_{i} \Pi_{j}\left(u_{j}^{k-1}(t)\right)\right) d t .
$$

Last, we check that the NL-OSWR algorithm is well posed. That is, we need to verify that Assumption 9 holds for every iteration. The initial iterate and the Robin coefficients are chosen such that it holds for $k=0$. We have been able to show that this remains true throughout the algorithm only in the matching case, that is when the capillary pressure functions satisfy

$$
\pi_{1}(0)=\pi_{2}(0) \text { and } \pi_{1}(1)=\pi_{2}(1) .
$$

\section{Numerical experiment}

The domain $\Omega$ is the unit cube, decomposed into two subdomains with two rock types (see figure 1 . The mobilities and capillary pressure functions are given by

$$
\lambda_{o, i}(u)=u, i \in\{1,2\}, \quad \pi_{1}(u)=5 u^{2}, \quad \text { and } \quad \pi_{2}(u)=5 u^{2}+1 .
$$

The initial condition is that the domain contains some quantity of gas, situated only within $\Omega_{1}$. The domain is discretized by a mesh of $20 \times 20 \times 20$ elements, the 
time discretization is non-conforming, with constant time steps in each subdomain $\delta t_{1}=10^{-3}$, and $\delta t_{2}=\frac{1}{8} 10^{-2}$.

The full discretization was carried out with a two-point finite volume scheme [5]. One has to introduce additional unknowns on the interface in order to discretize the Robin transmission conditions. The method was implemented with the Matlab Reservoir Simulation Toolbox [13]. The nonlinear subdomain problem is solved with Newton's method.

The only change required to the finite volume scheme to cope with a nonconforming time scheme is the projection of the right hand side of the transmission condition on the grid of the current subdomain. This is what makes the choice of a DG formalism important, together with a global in time DD method. The resulting scheme is non-conforming in time, and the equivalence with the physical transmission conditions no longer holds. The projections can be computed using an algorithm with linear complexity described in [7, 8].
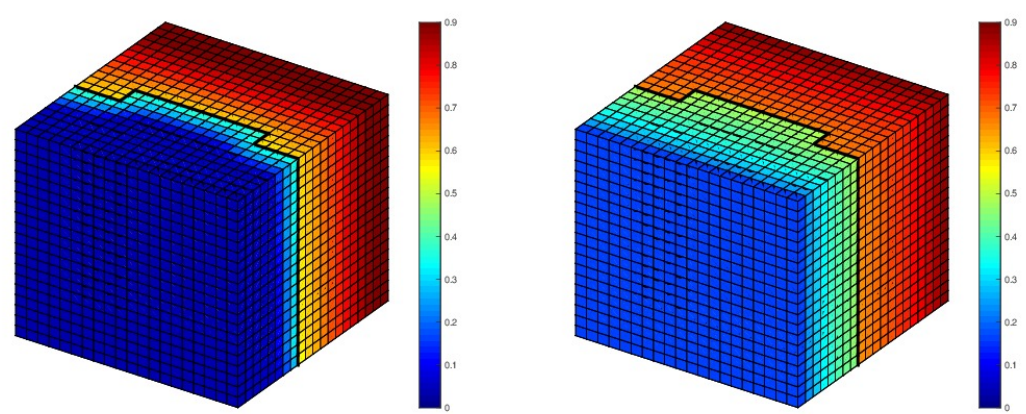

Fig. 1 Test case 1: Saturation $u(t)$ for $t=0.3$ and $t=3$

The evolution of the saturation at two time steps is shown in Fig. 1. We remark that at the beginning of the simulation, approximately until $t \approx 0.02$, the gas cannot penetrate to the domain $\Omega_{2}$, since the capillary pressure is lower than the threshold value $\pi_{2}(0)=1$, which is known as the entry pressure. The saturation of the trapped gas in $\Omega_{1}$ as well as the capillary pressure increase until the capillary pressure reaches the entry pressure.

We study the convergence behavior of the NL-OSWR algorithm. The tolerance for Newton's method is fixed to $10^{-8}$. The tolerance of the NL-OSWR algorithm is $10^{-6}$. The Robin parameters are chosen for the two subdomains so as to minimize the convergence rate of a linearized version of the problem. Precisely, we take in the model problem the capillary pressure as unknown, then linearize the nonlinear terms, leading to determine the optimal Robin parameters for a linear diffusion problem with discontinuous coefficients similar to that in [10, 12]. We show in Fig. 2 (right) the relative residuals comparing the convergence history with the parameters calculated numerically by minimizing the convergence factor for the linearized problem and 
that of with the best parameters located in the zone giving the smaller errors after the same number of iterations (see Fig. 2l left).
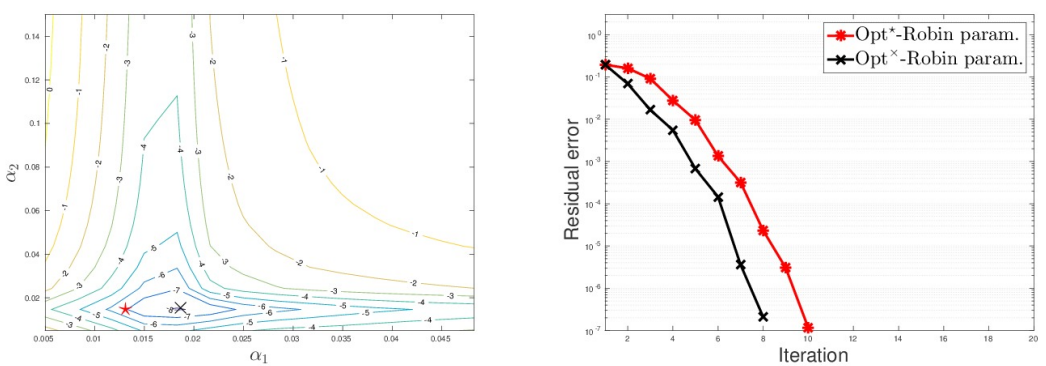

Fig. 2 Test case 1: Left: Level curves for the residual error obtained after 10 iterations for various values of the parameters $\alpha_{1}$ and $\alpha_{2}$. The star (in magenta) marked the parameters obtained with the minimization process of the convergence factor applied to the linearized problem which is close to the best one marked by times symbol (in black). Right: The convergence curves.

We now analyze the efficiency in time of the method with nonconforming time steps. We compute a reference solution as the converged multidomain solution with conforming fine time grids $\delta t_{f}=\frac{1}{4} 10^{-3}$, and where the relative residual is taken smaller than $10^{-12}$. We then compare the solution obtained with the nonconforming time steps, as described above with two solutions computed first with conforming fine time steps $\left(\delta t_{1}=\delta t_{2}=10^{-3}\right)$ and then with conforming coarse time steps $\left(\left(\delta t_{1}=\delta t_{2}=\frac{1}{8} 10^{-2}\right)\right)$. Fig. 3 shows the error in the saturation along a line orthogonal to the interface at three different time steps. One can see that the nonconforming solution as well as the solution with conforming and fine steps are in close agreement with the reference solution, whereas the solution with coarse time steps has a larger error. This confirms that nonconforming time grids with respect to the rock type numerically preserve the accuracy in time of the multidomain solution.
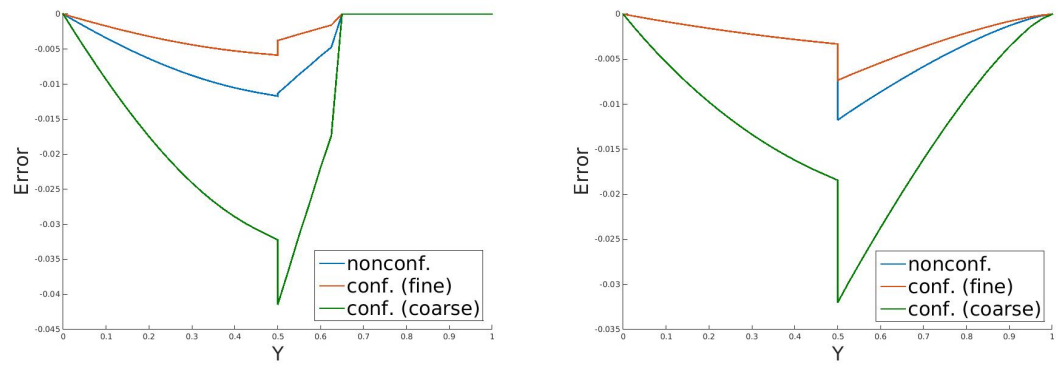

Fig. 3 Test case 1. Error in saturation along a line orthogonal to the interface, nonconforming and conforming (coarse and fine) time-steps. Left $T=T_{f} / 20$, right, $T=T_{f}$. 
Other examples with more physical content can be found in [1] and [2].

\section{Acknowledgments}

This work was funded by ANR DEDALES under grant ANR-14-CE23-0005

\section{References}

1. Ahmed, E., Hassan, S.A., Japhet, C., Kern, M., Vohralík, M.: A posteriori error estimates and stopping criteria for space-time domain decomposition for two-phase flow between different rock types. preprint HAL-01540956 (2017). Submitted for publication

2. Ahmed, E., Japhet, C., Kern, M.: A finite volume schwarz algorithm for two-phase immiscible flow with different rock types (2019). In preparation

3. Caetano, F., Gander, M.J., Halpern, L., Szeftel, J.: Schwarz waveform relaxation algorithms for semilinear reaction-diffusion equations. NHM 5(3), 487-505 (2010)

4. Cancès, C.: Nonlinear Parabolic Equations with Spatial Discontinuities. Nonlinear Differential Equations and Applications NoDEA 15(4-5), 427-456 (2008). DOI 10.1007/ s00030-008-6030-7

5. Enchéry, G., Eymard, R., Michel, A.: Numerical approximation of a two-phase flow problem in a porous medium with discontinuous capillary forces. SIAM Journal on Numerical Analysis 43(6), 2402-2422 (2006)

6. Ern, A., Mozolevski, I., Schuh, L.: Discontinuous Galerkin approximation of two-phase flows in heterogeneous porous media with discontinuous capillary pressures. Computer methods in applied mechanics and engineering 199(23), 1491-1501 (2010)

7. Gander, M.J., Japhet, C.: Algorithm 932: PANG: software for nonmatching grid projections in 2D and 3D with linear complexity. ACM Trans. Math. Software 40(1), Art. 6, 25 (2013). DOI $10.1145 / 2513109.2513115$

8. Gander, M.J., Japhet, C., Maday, Y., Nataf, F.: A New Cement to Glue Nonconforming Grids with Robin Interface Conditions: The Finite Element Case. In: T.J. Barth, M. Griebel, et al. (eds.) Domain Decomposition Methods in Science and Engineering, pp. 259-266. Springer Berlin Heidelberg, Berlin, Heidelberg (2005). DOI 10.1007/3-540-26825-1_24

9. Halpern, L., Japhet, C., Szeftel, J.: Optimized Schwarz waveform relaxation and discontinuous Galerkin time stepping for heterogeneous problems. SIAM J. Numer. Anal. 50(5), 2588-2611 (2012). DOI 10.1137/120865033

10. Hoang, T.T.P., Jaffré, J., Japhet, C., Kern, M., Roberts, J.E.: Space-time domain decomposition methods for diffusion problems in mixed formulations. SIAM Journal on Numerical Analysis 51(6), 3532-3559 (2013). DOI 10.1137/130914401

11. Hoang, T.T.P., Japhet, C., Kern, M., Roberts, J.E.: Space-time domain decomposition for advection-diffusion problems in mixed formulations. Mathematics and Computers in SImulation 137, 366-389 (2016). DOI 10.1016/j.matcom.2016.11.002

12. Lemarié, F., Debreu, L., Blayo, E.: Toward an optimized global-in-time schwarz algorithm for diffusion equations with discontinuous and spatially variable coefficients, part 1: the constant coefficients case. Electron. Trans. Numer. Anal 40, 148-169 (2013)

13. Lie, K.A., Krogstad, S., Ligaarden, I.S., Natvig, J.R., Nilsen, H.M., Skaflestad, B.: Open source MATLAB implementation of consistent discretisations on complex grids. Comput. Geosci. 16(2), 297-322 (2012). DOI 10.1007/s10596-011-9244-4

14. Van Duijn, C.J., Molenaar, J., De Neef, M.J.: The effect of capillary forces on immiscible twophase flow in heterogeneous porous media. Transport in Porous Media 21(1), 71-93 (1995). DOI 10.1007/BF00615335 